\title{
The Nobel Prize in Chemistry 2017: High-Resolution Cryo-Electron Microscopy
}

\author{
Jae-Hee Chung, Ho Min Kim ${ }^{1, *}$ \\ Department of Biological Sciences, Korea Advanced Institute of Science and Technology (KAIST), Daejeon 34141, Korea \\ ${ }^{1}$ Graduate School of Medical Science \& Engineering, Korea Advanced Institute of Science and Technology (KAIST), \\ Daejeon 34141, Korea
}

*Correspondence to:

Kim HM,

Tel: +82-42-350-4244

Fax: +82-42-350-8160

E-mail: hm_kim@kaist.ac.kr

Received November 15, 2017

Revised December 4, 2017

Accepted December 4, 2017
The 2017 Nobel Prize in Chemistry was awarded to the following three pioneers: Dr. Joachim Frank, Dr. Jacques Dubochet, and Dr. Richard Henderson. They all contributed to the development of a Cryo-electron microscopy (EM) technique for determining the highresolution structures of biomolecules in solution, particularly without crystal and with much less amount of biomolecules than X-ray crystallography. In this brief commentary, we address the major advances made by these three Nobel laureates as well as the current status and future prospects of this Cryo-EM technique.

Key Words: High-resolution Cryo-electron microscopy, Nobel Prize, Biomolecule structure
Since the structure of myoglobin was first determined in the 1950s by X-ray crystallography, more than 130,000 biomolecular structures, including protein domain, protein/ protein complex, protein/DNA complex, and protein/ RNA complex, have been deposited in the Protein Data Bank, which is a central repository for three-dimensional (3D) biomolecular structural data. The knowledge of these biomolecular structures has significantly advanced our understanding of biological phenomena at the molecular level and has facilitated pharmaceutical development. Despite the considerable success of X-ray crystallography in determining the structures of biomolecules, this method still has fundamental limitations. Large amounts of high purity proteins are required for crystallization and procuring adequate well-organized crystal, which is sufficient to determine the atomic structure, is sometimes very difficult. Therefore, many of macromolecular complex $(>100 \mathrm{kDa})$, polymerizing proteins, integral membrane proteins, and proteins with multiple conformations or with flexible domain have been awaiting the birth of new technology, highresolution CryoEM, for their structure analysis.

The invention of transmission electron microscopy (TEM) by Ernst Ruska in the 1930s was immediately welcomed by physicists and materials scientists owing to its wide range of applications. However, this was not the case for biologists mainly because of the intrinsic problems associated with biomolecules. In particular, biomolecules are sensitive to radiation damage, can be easily dehydrated under the high vacuum conditions required for TEM, and have poor electron scattering capability due to their low weight elemental composition. To overcome these issues, negative staining, which involves embedding a biomolecule in a layer of dried heavy metal solution, such as uranyl formate or uranyl acetate, was first employed in the 1940s and was further refined during the next 20 years (Brenner \& Horne, 1959; Hall et al., 1945; Huxley \& Zubay, 1961). This specimen preparation for TEM is quick and easy, and allows the collection of detailed information about the morphology of bacteria, viruses, organelles, and protein complexes. Nevertheless, staining with the heavy metal solution and drying often caused the structural collapse of biomolecules, and the resolution is limited by the granularity of heavy metal used for negative stain. Therefore, the development of an alternative sample preparation method that enables the study of unstained

(a) This is an open-access article distributed under the terms of the Creative Commons Attribution Non-Commercial License (http://creativecommons.org/licenses/by-nc/4.0) which permits unrestricted noncommercial use, distribution, and reproduction in any medium, provided the original work is properly cited.

Copyrights @ 2017 by Korean Society of Microscopy 
biomolecules was urgently needed.

In the mid-1970s, Richard Henderson and Nigel Unwin replaced water with a glucose solution to preserve an unstained bacteriorhodopsin sample in vacuum (Henderson \& Unwin, 1975). Moreover, they acquired TEM images using a very low electron dose to minimize electron radiation damage. An analysis of the resulting electron micrographs and the construction of a 3D map from $2 \mathrm{D}$ projections, that was based on the "central section theorem" developed by Aaron Klug et al. along with electron diffraction data generated from a native bacteriorhodopsin crystal, revealed the first bacteriorhodopsin structure at $\sim 7 \AA$ resolution (De Rosier \& Klug, 1968; Henderson \& Unwin, 1975). Unfortunately, Henderson's use of a glucose solution for membrane-bound bacteriorhodopsin was not generally applicable to other water-soluble proteins. Cooling the specimen was then employed to reduce the evaporation of water and to protect the specimen from radiation damage (Taylor \& Glaeser, 1974). However, upon freezing, water typically nucleates to form crystalline ice; this strongly diffracts electrons, thus effectively obliterating signals originated from the sample. Furthermore, the formation of ice crystals may change the structure of the specimen. Problems associated with the formation of crystalline ice were overcome with a vitrification technique developed by Jacques Dubochet (Dubochet \& McDowall, 1981; Dubochet et al., 1988). Rapidly plunging the specimen into a very low temperature cryogen, such as liquid ethane $\left(-190^{\circ} \mathrm{C}\right)$, creates a non-crystalline, glasslike solid form of water known as amorphous or vitreous ice. This allows the biomolecules to be preserved close to their native state in diverse orientations. In 1984, Jacques Dubochet and Marc Adrian published the first images of several viruses in vitrified ice; these viruses showed a sharp contrast and were devoid of damage resulting from sample preparation (Adrian et al., 1984). Using this elaborate sample vitrification technique along with image acquisition at cryogenic temperatures, Henderson finally determined 3.5 A structure of bacteriorhodopsin in 1990 (Henderson et al., 1990). This groundbreaking work demonstrated for the first time, that high-resolution structures of biomolecules could be obtained using Cryo-EM through averaging over many copies of the same object. Henderson made the following argument, "This would then turn the technique we have been using into a routine and quick method, able to be used on many more difficult specimens, eventually including non-crystalline molecular assemblies."

For non-periodic assemblies of symmetrical particles, the signal-to-noise ratio can be improved by averaging the asymmetric units. However, for non-crystalline, asymmetric, and unstained biomolecules, discriminating the position and orientation of each particle from the background with such a low contrast micrograph is challenging. Moreover, biomolecules may contain impurities and have different conformations. Therefore, it is also necessary to identify potential structural sub-states and to identify different types of particles within heterogeneous particles in a micrograph. Joachim Frank addressed these issues with a theoretical method for "single particle analysis," which involved multivariate statistical analysis and a projection matching algorithm, and implemented this method on a computer software known as System for Processing of Image Data in Electron microscopy and Related fields (SPIDER) in 1981 (Frank \& van Heel, 1982; Frank et al., 1981; Radermacher et al., 1986; Shaikh et al., 2008; van Heel \& Frank, 1981). Numerous other imaging programs such as EMAN2 (Tang et al., 2007), XMIPP (Scheres et al., 2008), IMAGIC (van Heel et al., 1996), FREALIGN (Grigorieff, 2007), and RELION (Scheres, 2012) are now available. However, it is reasonable to say that the computational aspect of Cryo-EM technology was initiated and established from Joachim Frank's work. Overall, the work of Richard Henderson, Joachim Frank, and Jacques Dubochet represented the advent of Cryo-EM technology.

Despite such landmark advances by the three scientists who became Nobel laureates in chemistry in 2017, it was not until the 2010s that we reached the true era of highresolution Cryo-EM. Many technical innovations, such as the field emission gun, stable cold stage, $\mathrm{Cs} / \mathrm{Cc}$ corrector, energy filter, and direct electron detector, enable CryoEM to reach unprecedented atomic resolution, which is comparable to the results of X-ray crystallography. In particular, the direct electron detector, which is the most critical breakthrough for high-resolution Cryo-EM, can detect electron signals at low intensities with reduced noise, and its fast read-out speed allows a "movie" to be recorded (Bai et al., 2015; Li et al., 2013). This movie mode acquisition was able to computationally correct beam-induced particle movement; thus, it could remove blurring and dramatically improve the image quality. Many opportunities remain to in the field of Cryo-EM. These include even more improved detectors and phase plate technology (Danev et al., 2014), increased reproducibility of cryo-EM sample preparation, and software based on a new algorithm that can efficiently handle the structural heterogeneity of samples with reduced computational load. Many of these have been investigated by Cryo-EM communities and hope to be released in the near future.

Although the structure determination of purified macromolecular biomolecules via single particle Cryo-EM has recently received much attention (Fig. 1; Jeong et al., 2017), in situ structural biology involving Cryo-electron tomography, which allows molecular assemblies and machines to be studied in an in situ cellular environment, may lead to the next revolution in Cryo-EM technology. Electron tomography is a method for reconstructing $3 \mathrm{D}$ tomogram 


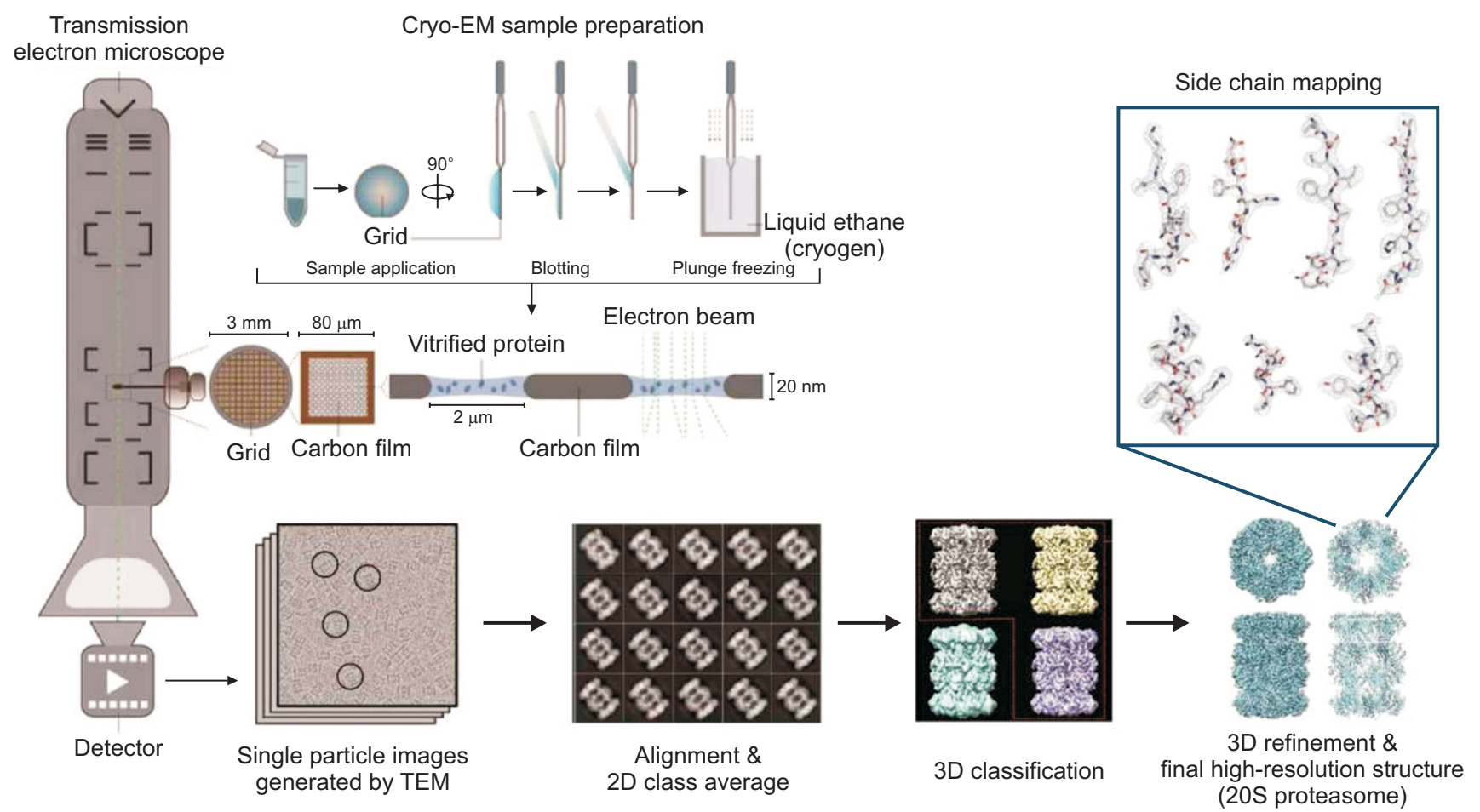

Determination of high-resolution structure through image processing

Fig. 1. Cryo-electron microscopy (EM) Work Flow. A purified sample is applied to the grid and then vitrified with liquid ethane. Particles embedded in the thin ice will have various random orientations, which are imaged by transmission electron microscopy (TEM) followed by motion correction and contrast transfer function (CTF) determination/correction. Individual particles are selected and aligned for two-dimensional (2D) class average. Threedimensional (3D) classification and further iterative refinement of 3D reconstruction will finally provide the high-resolution cryo-EM structure (3 Å cryoEM structure of archaea 20S proteasome processed with RELION 2.0) (Jeong et al., 2017).

by aligning and merging tilt series images that are acquired at different angular orientations of a sample. To improve the signals and resolution, repetitive molecular features in a 3D tomogram can be further extracted computationally and subjected to averaging and classification using methods similar to those employed in single particle analysis (Asano et al., 2016; Beck \& Baumeister, 2016). These methods, called subtomogram averaging, provided valuable insights into biological phenomena such as HIV maturation (Mattei et al., 2016; Schur et al., 2014, 2016), nuclear pore structures (Beck \& Glavy, 2014; Beck et al., 2007; Bui et al., 2013), coat protein complex I (Dodonova et al., 2015, 2017), and ribosometranslocation machinery (Pfeffer et al., 2015). However, a major drawback of these Cryo-electron tomography and subtomogram averaging technique is their limited resolution. Along with the technical innovation described above, further improvements, including focused ion beam micromachining to prepare samples with ideal thicknesses (Rigort et al., 2012; Villa et al., 2013), correlative light microscopyelectron microscopy (CLEM) (de Boer et al., 2015), and image processing algorithms such as denoising, template matching, and subtomogram averaging, as well as integration of complementary information on protein stoichiometry, proximity, and conformational variability with a highresolution structural repertoire contained in a database may enable Cryo-electron tomography to be developed as an unbiased discovery tool, namely in visual proteomics (Beck \& Baumeister, 2016; Nickell et al., 2006).

\section{CONFLICT OF INTEREST}

No potential conflict of interest relevant to this article was reported.

\section{ACKNOWLEDGMENTS}

This work was supported by grants from the National Research Foundation of Korea (NRF), Ministry of Science, ICT and Future Planning, Korea (NRF-2015R1A2A2A01005533). 


\section{REFERENCES}

Adrian M, Dubochet J, Lepault J, and McDowall A W (1984) Cryo-electron microscopy of viruses. Nature 308, 32-36.

Asano S, Engel B D, and Baumeister W (2016) In situ Cryo-electron tomography: a post-reductionist approach to structural biology. J. Mol. Biol. 428, 332-343.

Bai X C, McMullan G, and Scheres S H (2015) How cryo-EM is revolutionizing structural biology. Trends Biochem. Sci. 40, 49-57.

Beck M and Baumeister W (2016) Cryo-electron tomography: can it reveal the molecular sociology of cells in atomic detail? Trends Cell Biol. 26, 825-837.

Beck M and Glavy J S (2014) Toward understanding the structure of the vertebrate nuclear pore complex. Nucleus 5, 119-123.

Beck M, Lučić V, Förster F, Baumeister W, and Medalia O (2007) Snapshots of nuclear pore complexes in action captured by cryoelectron tomography. Nature 449, 611.

Brenner S and Horne R W (1959) A negative staining method for high resolution electron microscopy of viruses. Biochim. Biophys. Acta 34, 103-110.

Bui K H, von Appen A, DiGuilio A L, Ori A, Sparks L, Mackmull M T, Bock T, Hagen W, Andrés-Pons A, Glavy J S, and Beck M (2013) Integrated structural analysis of the human nuclear pore complex scaffold. Cell 155, 1233-1243.

Danev R, Buijsse B, Khoshouei M, Plitzko J M, and Baumeister W (2014) Volta potential phase plate for in-focus phase contrast transmission electron microscopy. Proc. Natl. Acad. Sci. U S A 111, 15635-15640.

de Boer P, Hoogenboom J P, and Giepmans B N G (2015) Correlated light and electron microscopy: ultrastructure lights up! Nat. Meth. 12, 503-513.

De Rosier D J and Klug A (1968) Reconstruction of three dimensional structures from electron micrographs. Nature 217, 130-134.

Dodonova S O, Aderhold P, Kopp J, Ganeva I, Rohling S, Hagen W J H, Sinning I, Wieland F, and Briggs J A G (2017) 9A structure of the COPI coat reveals that the Arf1 GTPase occupies two contrasting molecular environments. Elife 6.

Dodonova S O, Diestelkoetter-Bachert P, von Appen A, Hagen W J H, Beck R, Beck M, Wieland F, and Briggs J A G (2015) A structure of the COPI coat and the role of coat proteins in membrane vesicle assembly. Science 349, 195.

Dubochet J, Adrian M, Chang J J, Homo J C, Lepault J, McDowall A W, and Schultz P (1988) Cryo-electron microscopy of vitrified specimens. Q. Rev. Biophys. 21, 129-228.

Dubochet J and McDowall A W (1981) Vitrification of pure water for electron microscopy. J. Microsc. 124, RP3-RP4.

Frank J, Shimkin B, and Dowse H (1981) SPIDER - a modular software system for electron image processing. Ultramicroscopy 6, 343-358.

Frank J and van Heel M (1982) Correspondence analysis of aligned images of biological particles. J. Mol. Biol. 161, 134-137.

Grigorieff N (2007) FREALIGN: high-resolution refinement of single particle structures. J. Struct. Biol. 157, 117-125.

Hall C E, Jakus M A, and Schmitt F O (1945) The structure of certain muscle fibrils as revealed by the use of electron stains. J. Appl. Phys. 16, 459-465.

Henderson R, Baldwin J M, Ceska T A, Zemlin F, Beckmann E, and
Downing K H (1990) Model for the structure of bacteriorhodopsin based on high-resolution electron cryo-microscopy. J. Mol. Biol. 213, 899-929.

Henderson R and Unwin P N (1975) Three-dimensional model of purple membrane obtained by electron microscopy. Nature 257, 28-32.

Huxley H E and Zubay G (1961) Preferential staining of nucleic acidcontaining structures for electron microscopy. J. Biophys. Biochem. Cytol. 11, 273-296.

Jeong H, Lee S G, Kweon H S, and Hyun J (2017) Facility for high resolution cryo-electron microscopy of biological macromolecules at Korea Basic Science Institute. Biodesign 5, 96-102.

Li X, Mooney P, Zheng S, Booth C R, Braunfeld M B, Gubbens S, Agard D $A$, and Cheng $Y$ (2013) Electron counting and beam-induced motion correction enable near-atomic-resolution single-particle cryo-EM. Nat. Methods 10, 584-590.

Mattei S, Glass B, Hagen W J H, Kräusslich H G, and Briggs J A G (2016) The structure and flexibility of conical HIV-1 capsids determined within intact virions. Science 354, 1434.

Nickell S, Kofler C, Leis A P, and Baumeister W (2006) A visual approach to proteomics. Nat. Rev. Mol. Cell Biol. 7, 225.

Pfeffer S, Burbaum L, Unverdorben P, Pech M, Chen Y, Zimmermann R, Beckmann R, and Forster F (2015) Structure of the native Sec61 protein-conducting channel. Nat. Commun. 6, 1632-1643.

Radermacher M, Wagenknecht T, Verschoor A, and Frank J (1986) A new 3-D reconstruction scheme applied to the $50 S$ ribosomal subunit of $E$. coli. J. Microsc. 141, RP1-2.

Rigort A, Bäuerlein F J B, Villa E, Eibauer M, Laugks T, Baumeister W, and Plitzko J M (2012) Focused ion beam micromachining of eukaryotic cells for cryoelectron tomography. Proc Natl. Acad. Sci. 109, 44494454.

Scheres S H (2012) RELION: implementation of a Bayesian approach to cryo-EM structure determination. J. Struct. Biol. 180, 519-530.

Scheres S H, Nunez-Ramirez R, Sorzano C 0, Carazo J M, and Marabini R (2008) Image processing for electron microscopy single-particle analysis using XMIPP. Nat. Protoc. 3, 977-990.

Schur F K M, Hagen W J H, Rumlová M, Ruml T, Müller B, Kräusslich H G, and Briggs J A G (2014) Structure of the immature HIV-1 capsid in intact virus particles at $8.8 \AA$ resolution. Nature 517, 505.

Schur F K M, Obr M, Hagen W J H, Wan W, Jakobi A J, Kirkpatrick J M, Sachse C, Kräusslich H G, and Briggs J A G (2016) An atomic model of HIV-1 capsid-SP1 reveals structures regulating assembly and maturation. Science 353, 506.

Shaikh T R, Gao H, Baxter W T, Asturias F J, Boisset N, Leith A, and Frank $J$ (2008) SPIDER image processing for single-particle reconstruction of biological macromolecules from electron micrographs. Nat. Protoc. 3, 1941-1974.

Tang G, Peng L, Baldwin P R, Mann D S, Jiang W, Rees I, and Ludtke S $J$ (2007) EMAN2: an extensible image processing suite for electron microscopy. J. Struct. Biol. 157, 38-46.

Taylor K A and Glaeser R M (1974) Electron diffraction of frozen, hydrated protein crystals. Science 186, 1036-1037.

van Heel M and Frank J (1981) Use of multivariate statistics in analysing the images of biological macromolecules. Ultramicroscopy $\mathbf{6 , 1 8 7 -}$ 194. 
van Heel M, Harauz G, Orlova E V, Schmidt R, and Schatz M (1996) A new generation of the IMAGIC image processing system. J. Struct. Biol. 116, 17-24.
Villa E, Schaffer M, Plitzko J M, and Baumeister W (2013) Opening windows into the cell: focused-ion-beam milling for cryo-electron tomography. Curr. Opin. Struct. Biol. 23, 771-777. 